\title{
Research on the Influence of Load Characteristics of Distributed Power Grid on Electric Energy Metering
}

\author{
Li Pengcheng ${ }^{1}$, Zhao Yantao ${ }^{2}$, Cong Zhongxiao ${ }^{1}$, Zhao Yunbin $^{1}, \mathrm{Xu}_{\text {Hongwei }}{ }^{1}, \mathrm{Su}_{\text {Yuhua }}{ }^{2}$ \\ ${ }^{1}$ Guizhou Power Grid Co., Ltd., Guiyang, China \\ ${ }^{2}$ Wasion Group Co., Ltd., Changsha, China
}

Email address:

365248318@qq.com (Li Pengcheng),644397824@qq.com (Cong Zhongxiao),28609956@qq.com (Zhao Yunbin), 275853249@qq.com (Xu Hongwei),suyuhua@wasion.com (Su Yuhua), zhaoyantao@wasion.com (Zhao Yantao)

\section{To cite this article:}

Li Pengcheng, Zhao Yantao, Cong Zhongxiao, Zhao Yunbin, Xu Hongwei, Su Yuhua. Research on the Influence of Load Characteristics of Distributed Power Grid on Electric Energy Metering. Journal of Electrical and Electronic Engineering. Vol. 6, No. 3, 2018 , pp. 94-103. doi: $10.11648 /$ j.jeee.20180603.15

Received: July 6, 2018; Accepted: August 16, 2018; Published: September 15, 2018

\begin{abstract}
As a large number of Distributed Generation Connecting Electric Grid put into operation, there are many impacts on power metering of power grid due to the problems of large fluctuation of load and frequent reverse of power, injecting DC component and harmonic component into power grid, generating voltage deviation, voltage fluctuation and flicker, and voltage unbalance. Measurement accuracy of traditional metering devices is difficult to guarantee. Therefore, the adaptability of traditional metering devices in the distributed power grid environment and the improvement of new technical methods to the Distributed Generation Connecting Electric Grid need to be studied. In this paper, through the analysis of the field operation data, it is proposed that the load fluctuation and frequent reverse of power can be solved by using the bidirectional and wide-range metering gateway meter; by analyzing the influence of the secondary transformer on the DC component, it is proposed that the influence of the DC component on the power metering can be almost ignored under the $0.5 \%$ DC component injection of the Distributed Generation Connecting Electric Grid. By analyzing zero flux technology, phase matching technology and high frequency sampling technology, a method to solve the problem of synchronous sampling of voltage and current under the influence of harmonics is proposed. By analyzing the point product and harmonic algorithm, the sampling rate requirement of accurate measurement of electric energy is proposed. A series of reasonable technical means and requirements are put forward in this paper, which greatly improves the power metering problem under the Distributed Generation Connecting Electric Grid.
\end{abstract}

Keywords: Distributed Power, Wide Range, DC Component, Harmonics, Power Factor, Energy Metering

\section{Foreward}

Distributed power supply refers to the resources located in the vicinity of the load, with a small installed capacity, and being connected to the medium- and low-voltage distribution network nearby. The distributed power is divided into distributed power generation and energy storage, mainly including photovoltaic, wind power, hydropower, biomass and other renewable energy power generation and gas turbines, internal combustion engines and other clean energy power generation equipment. With a large number of distributed power supplies connected to the grid, the distributed power supply has improved the energy supply structure to a certain extent, but a large number of studies have shown that due to the large amount of impurities in the grid connected to distributed power, the electricity generated has a lot of influence on the quality of the grid energy [1-2], such as the injection of DC components and harmonic components, voltage deviation, voltage fluctuations and flicker and voltage imbalance, etc. [3-5], distributed power supply grid-connected also has the problem of bidirectional wide range measurement [6-8]. The state has issued a number of grid-connected technical standards to limit the distributed power grid-connected technical indicators to reduce the negative impact of grid power quality [9-12]. Among them, "Q/GDW 480-2010 Technical rule for distributed resources connected to power grid" requires: 1) When the converter type is a distributed power grid rated operation, the DC current component fed to the grid should not exceed $0.5 \%$ of its AC 
fixed value; 2) The harmonic current component 1 to 25 degrees (rms value) of the common connection point connected to the distributed power should meet the requirements of GB/T 14549-1993 "Quality of electric energy supply Harmonics in supply network"[13]. Because the power quality of the distributed power grid point is affected, involving the settlement of benefits between the power supply and consumption, it is necessary to study the metering device adapted to the distributed power grid load characteristics. In this article, the high- and medium-voltage distributed power grid is taken as the research object, and the influence of measuring of the wide-range measurement, the DC component, the harmonic component and the power factor on the metering is studied separately.

\section{Research on Bidirectional Wide Range Measurement Technology}

\subsection{Bidirectional Wide Range Characteristics and Measuring Point Settings}

(1) Bi-directional power flow wide-range measuring load characteristics

Due to the intermittent and randomness of distributed power sources, it is impossible for it to be used as a power source to generate electricity, but rather to be both a power source and an electrical load. As a power generation user, with large power of power generation, and power transmission is power to the grid. As a power user, a distributed power needs to draw power from the grid in order to maintain the operation of the equipment. At this time, the required power is very small, and the power transmission is from the power grid to the power. Comparing distributed power generation with power load conditions, it can be concluded that the distributed power supply has a large load change at the grid point, and the maximum power variation range is the maximum power generation PMAX/minimum load operating power PMIN of the user, and there are forward and reverse power flows.

When a distributed power source is used as a user, reactive power compensation should be carried out rationally with its load and voltage fluctuations. No compensation or over-compensation is not conducive to economical operation of the power grid. In order to operate economically and reliably, the reactive power compensation device should not be able to control inductive reactive power and capacitive reactive power. In the four-quadrant reactive power combination measuring, as a user, the reactive power needs to be combined in perceptual reactive power and capacitive reactive power. This combination mode is just in line with the purpose of improving the power.

(2) Distributed power grid measuring point settings

There are two ways to set a distributed power grid measuring point [8], single meter metering and double meter metering. Single meter metering is to connect the meter before the power load, as shown in Figure 1. When a distributed power source generates power, the power meter measures power generation; when the power load is used to draw power from the power grid, the power meter measures power consumption. This method requires that the energy meter should be able to accurately measure forward and reverse power, with a wide range of measuring capabilities. Dual metering is to connect the energy meter to the position 2 and 3, respectively. The meter of position 2 can measure the total power generated by the distributed power, and the meter of position 3 can measure the power consumption of user load. This method does not require the energy meter to have a bi-directional wide-range measuring function, but at the expense of a multi-meter. When the distributed power system has a large structure, a large number of power meters are required for such a mode and resources are wasted.

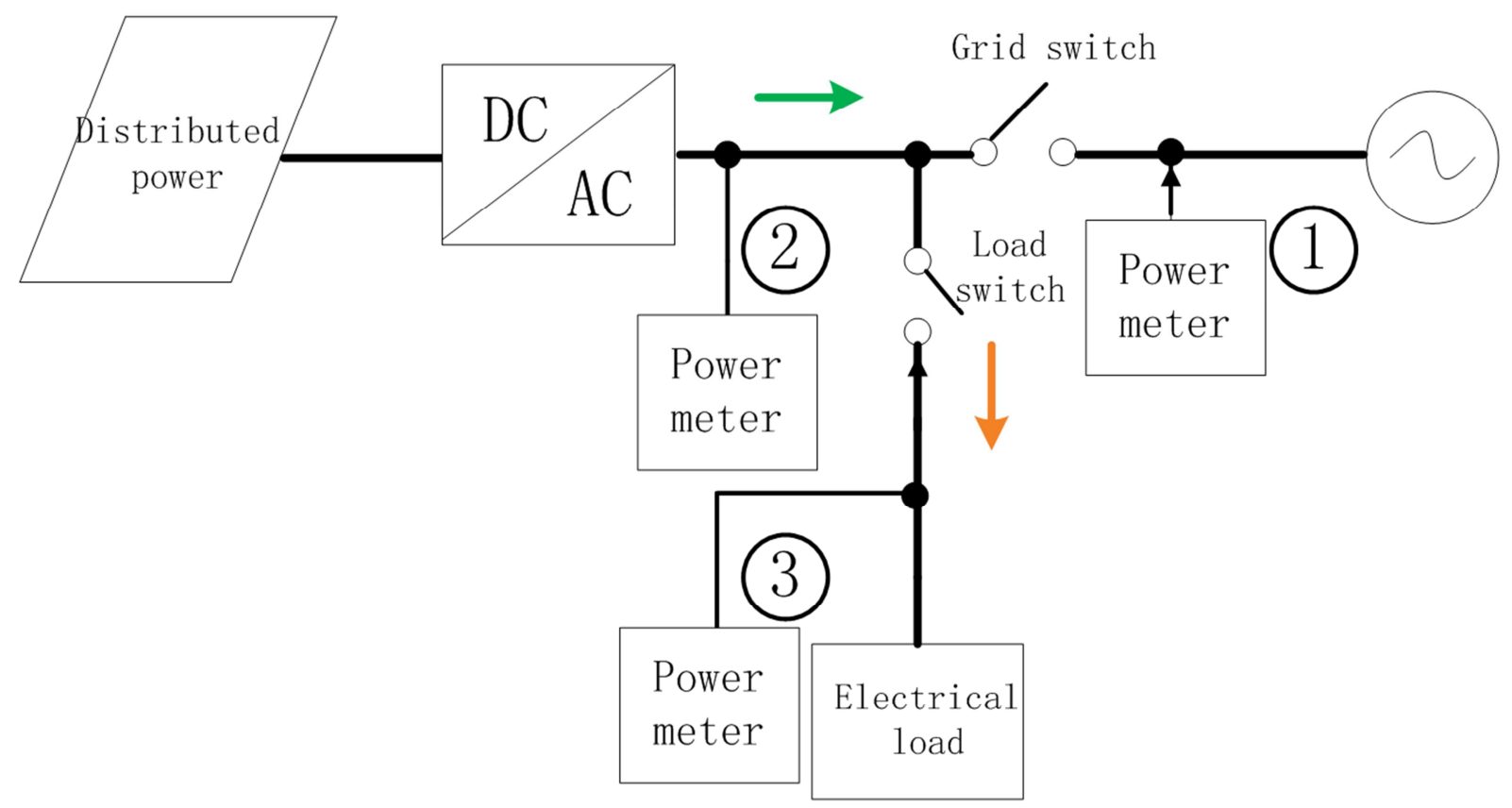

Figure 1. Distributed power grid-connected measuring point setting. 


\subsection{Distributed Power on-Site Problem Analysis}

Take the gateway of $220 \mathrm{kV} / 600 \mathrm{~A}$ wind farm at Bayannur Electric Power Bureau as an example to analyze the problem of dual wide-range measuring of distributed power. The structure of the wind farm system is shown in Figure 2.
To hang a factory gateway power meter A (wide range measuring) with specification of 0.3 (6) A and an import meter B (ordinary metering) with a specification of 5 (6) A at the metering point of the gateway of the same wind farm, and the results of comparison after several months are shown in Table 1.

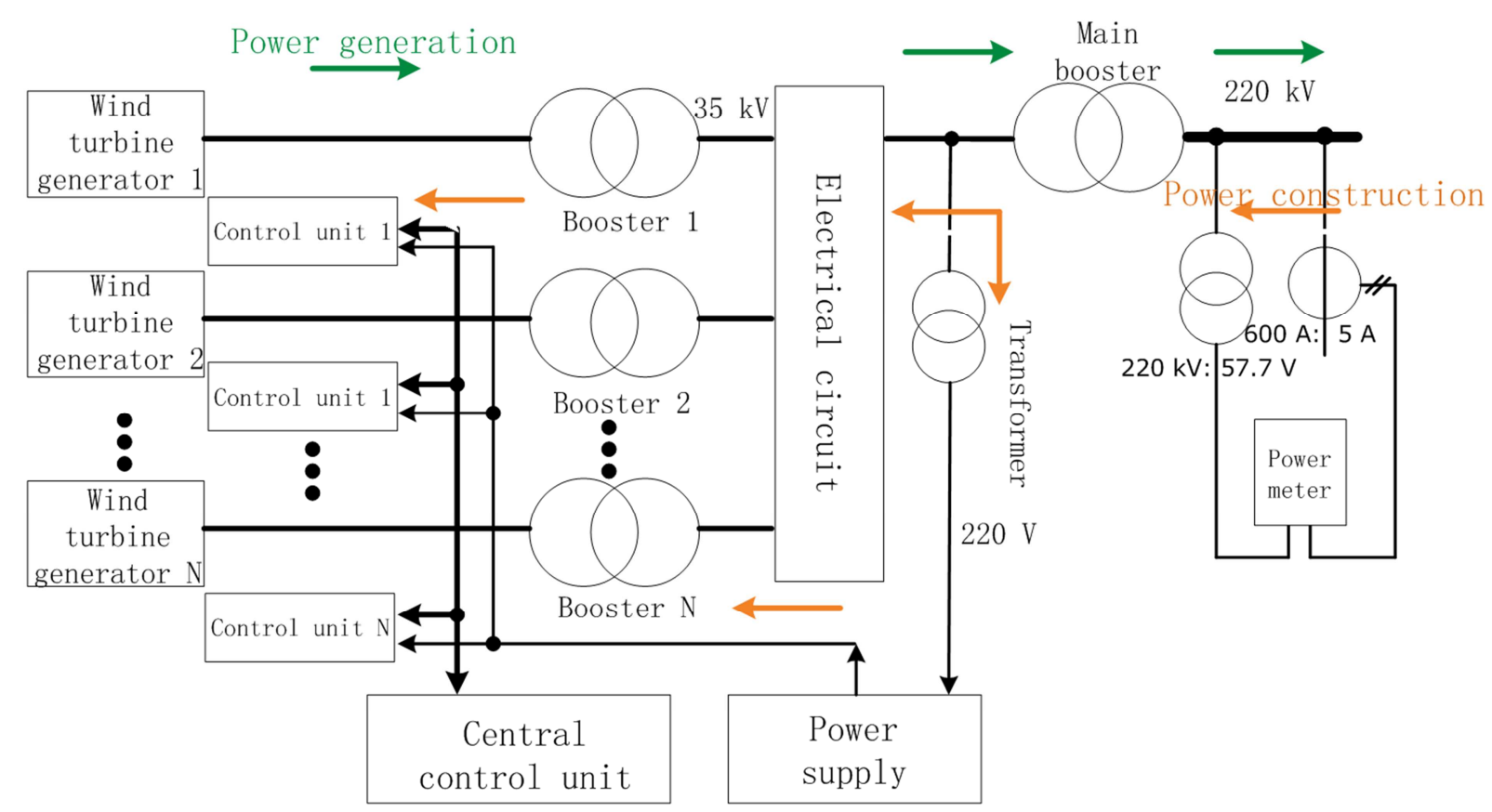

Figure 2. System structure diagram of wind farm gateway.

Table 1. Operation data of wind farm gateway.

\begin{tabular}{llllll}
\hline Line number & Meter type & Active meter bottom & Rate & Power & Difference/(\%) \\
\hline & A factory gateway meter A consumption network & 0.812 & 1.693 & 264000 & 232584 \\
& A factory gateway meter A generation network & 194.682 & 313.949 & 264000 & 31486488 \\
& An import meter B consumption network & 1997.1 & 2208.35 & 1000 & 211250 \\
& An import meter B generation network & 410504.16 & 441972.36 & 1000 & 31468200 \\
Power difference & Consumption network: 232 584-211 250=21 334 (kWh) & & \\
analysis & Generation network: 31 486 488-31 468 200=18 288 (kWh) & & \\
\hline
\end{tabular}

When the wind farm generates electricity to the grid, no matter whether it is a import meter of specification 5 (6) A or a meter of specification 0.3 (6) A, it has good measurement accuracy in the operating current range, and the difference in forward energy is very small.

When the wind farm absorbs energy to the grid, it is mainly used for the work needs of some functional components inside the fan, such as work of the yaw, pitch changes, and electric control cabinet. The power consumed by these features before they are connected to the grid is different for each fan. For example, some fans may need to yaw before they are connected to the grid, and other units may be facing the wind direction before connecting to the grid, and no yawing is required. In general, the power consumption of the fan before it is connected to the grid is between 0 and $20 \mathrm{~kW}$, with an average of about $8 \mathrm{~kW}$.

Calculated with a single fan startup power of $8 \mathrm{~kW}$, starting consumption current converted to $220 \mathrm{kV}$ side corresponding fan is:

$$
I_{1}=8 \mathrm{~kW} / 220 \mathrm{kV}=36.36 \mathrm{~mA}
$$

After the current is transformed by the current transformer, the corresponding consumption current into the meter is:

$$
I_{1}=36.36 \mathrm{~mA} / 600 \mathrm{~A} * 5 \mathrm{~A}=0.303 \mathrm{~mA}
$$

The starting current of the 5 (6) A energy meter is $5 \mathrm{~mA}$, which means that when 16 fans or less are started at the same time, the power obtained from the power grid cannot be metered, resulting in reverse power loss;

The 0.3 (6) A power meter has a starting current of $0.3 \mathrm{~mA}$, which means that even if only one fan is started, the energy consumed can also be measured.

Seen from the operating data, within the allowable error limits of the meter, the difference between the forward energy metering results of meter A and B is very small; however, the difference in reverse energy metering results is very large, close to $10 \%$. Obviously, the A meter has reduced the loss of 
reverse energy metering to a great extent due to its wide range measuring characteristics.

\section{Analysis of Influence of DC Component on Current Transformer}

The current domestic power meter generally adopts resistive voltage divider voltage sampling. Because the resistive divider is a linear element, its DC characteristic is better and it is influenced small by the DC. The influence of the loaded distributed power grid of current transformer when being injected into the DC component is analyzed below.
A large number of studies have shown that when the magnitude of the DC component is insufficient to cause magnetic saturation of the transformer, the power is hardly affected by the DC component. When the DC signal causes the core saturation of the current transformer, it will cause the electric quantity to be inaccurate. At this time, the current waveform will be distorted after being sampled by the transformer, and usually exists in the form of an even harmonic current [14-15]. For the three-phase four-wire connection mode, the total power of the fundamental wave of the power metering device is:

$$
\begin{aligned}
& P=P_{\mathrm{A}}+P_{\mathrm{B}}+P_{\mathrm{C}}=U_{\mathrm{A}}\left(\frac{f_{\mathrm{A}} \% I_{1 \mathrm{~A}}}{100}+I_{1 \mathrm{~A}}\right) \operatorname{Cos}\left(\theta-\delta_{\mathrm{A}}\right)+ \\
& U_{\mathrm{B}}\left(\frac{f_{\mathrm{B}} \% I_{1 \mathrm{~B}}}{100}+I_{1 \mathrm{~B}}\right) \operatorname{Cos}\left(\theta-\delta_{\mathrm{B}}\right)+U_{\mathrm{C}}\left(\frac{f_{\mathrm{C}} \% I_{1 \mathrm{C}}}{100}+I_{1 \mathrm{C}}\right) \operatorname{Cos}\left(\theta-\delta_{\mathrm{C}}\right)
\end{aligned}
$$

In the formula, $f_{\mathrm{A}} \% 、 f_{\mathrm{B}} \% 、 f_{\mathrm{C}} \% 、 \delta_{\mathrm{A}} 、 \delta_{\mathrm{B}} 、 \delta_{\mathrm{C}}$ represents the ratio difference and angle difference of current transformers in A, B and C phases, respectively. Because the DC bias will cause the change of ratio difference and angle difference of the current transformer [16], it will lead to deviation of the energy metering: When the ratio difference increases or the angle difference increases positively, the amount of electric energy is increased, and when the angle difference is increased negatively, the amount of electric energy is reduced.

The following uses a power meter with a current transformer as an example to add different sized DC signals to the current point of the current loop 0.5IMAX. The experimental data on the influence on power is shown in Figure 3:

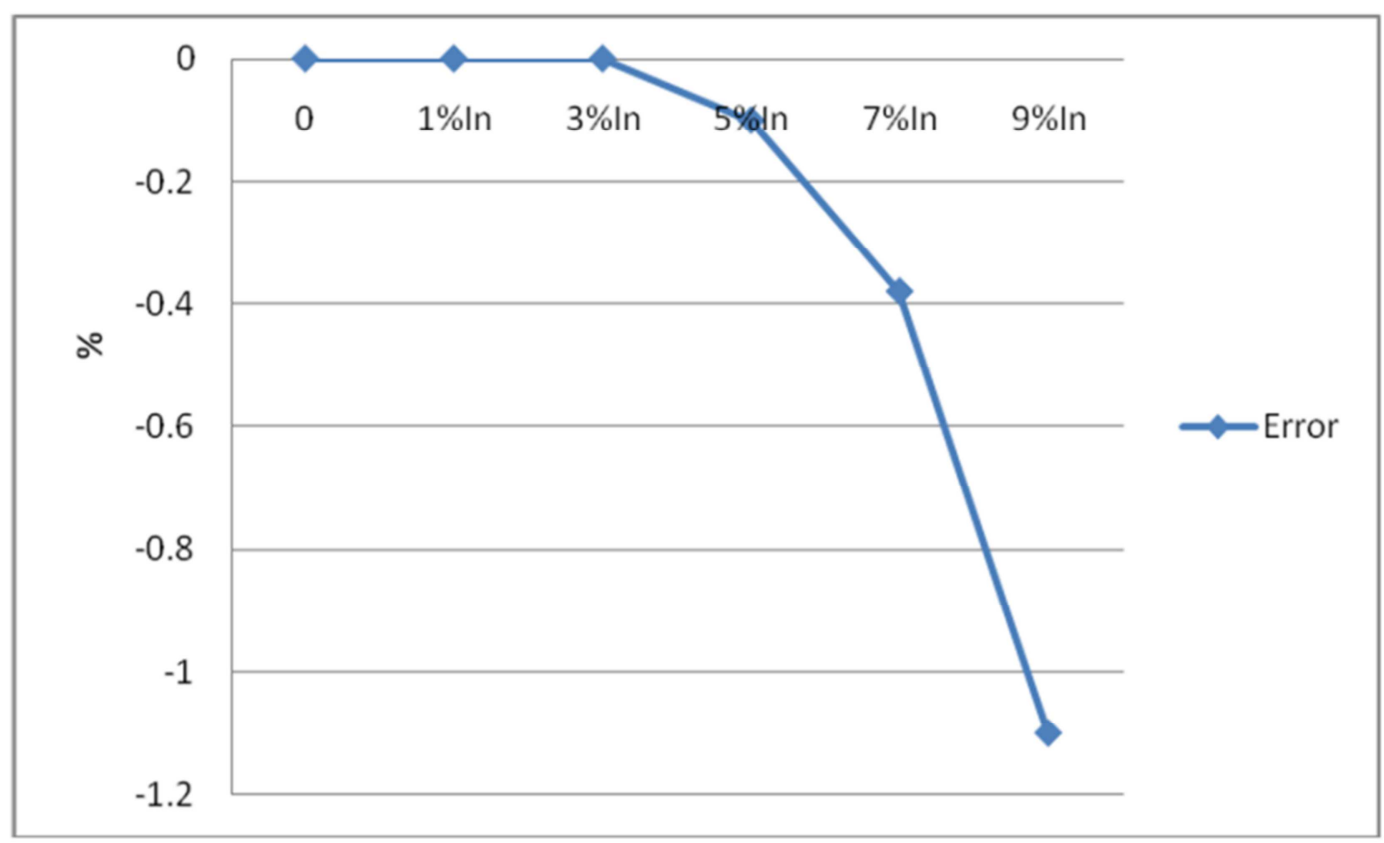

Figure 3. Power error under different DC components.

It can be seen from the data that when the DC component is less than $3 \%$, there is no effect on power. As the DC content increases, its power error increases rapidly. Because the DC component injected to the distributed power grid does not exceed $0.5 \%$ of its AC rating, which is much less than $3 \%$. Therefore, we can conclude that the impact of injection of DC components by distributed power on power metering is almost negligible.

\section{Analysis of Power Metering Under the Influence of Grid Harmonics}

Distributed power are often connected to the end of the grid feeder, which can easily cause voltage fluctuations, flicker, and dynamic and rapid changes in harmonic content [17-18]. Inverter's own modulation, dead band and other 
factors also produce harmonic currents. The power generation system composed of distributed power supplies has inherent harmonic resonance characteristics: its own internal resonance, parallel resonance between inverters, and series resonance between the power farm and the grid, making the harmonic current near the resonance point significantly amplified under certain conditions, and harmonic voltage and unbalanced three-phase of the power grid will also cause the PV inverter to generate harmonic currents of different times.

\subsection{Distributed Power Grid Harmonic Analysis}

In the pure sine signal, the instantaneous value of the voltage and current can be multiplied and then accumulate according to time to accurately measure the power of the signal. This method is called dot product sum algorithm. In the case of harmonics generated by the distributed power supply at the grid point, whether the harmonic measuring algorithm can accurately measure each harmonic component remains to be verified.

\subsubsection{Harmonic Measuring Algorithm Analysis}

For a signal containing N-degree harmonics, the angel frequency is $\omega_{n}$, then voltage and current waveforms can be expressed as:

$$
\begin{gathered}
u(t)=\sum_{k=0}^{M} u_{k}(t)=\sum_{k=0}^{M} u_{\mathrm{m} k} \sin \left(\omega_{k} t+\theta_{k \mathrm{u}}\right) \\
\mathrm{P}=\mathrm{UI \operatorname {Icos } ( \theta )}
\end{gathered}
$$

Where $\mathrm{M}$ is the degree of harmonics, $\delta$ and $\mathrm{P}_{m}=U I \cos (\theta+\delta)$ are the maximum value of the kth harmonic voltage and current, respectively, $\theta_{k \mathrm{u}}$ and $\theta_{k \mathrm{i}}$ are their initial phase angles. The active power $\mathrm{P}$ is:

$$
P=\frac{1}{T} \int_{0}^{T} u(t) i(t) \mathrm{d} t=\frac{1}{T} \int_{0}^{T} p(t) \mathrm{d} t
$$

After discretizing the voltage and current waveforms, there are:

$$
\mathrm{P}=\frac{1}{N} \sum_{n=1}^{N} u(n) i(n)
$$

The result of the dot product sum of the voltage and current instantaneous value is:

$$
\begin{aligned}
\mathrm{P}_{1 \mathrm{~N}}= & \frac{1}{N} \sum_{d=0}^{N-1} u_{d}(t) i_{d}(t)= \\
& \frac{U_{m n} I_{m n}}{N} \sum_{d=0}^{N-1} \sin \left(\frac{2 \pi}{N} \cdot d+\theta_{a n}\right) \sin \left(\frac{2 \pi}{N} \cdot d+\theta_{b n}\right)
\end{aligned}
$$

The $\mathrm{P}_{1 \mathrm{~N}}$ obtained when $\mathrm{N}$ takes different values are:

$$
\begin{gathered}
P_{11}=U_{\mathrm{m} n} \mathrm{I}_{\mathrm{m} n} \sin \theta_{\mathrm{a} n} \sin \theta_{\mathrm{b} n}=\frac{1}{2} U_{\mathrm{m} n} \mathrm{I}_{\mathrm{m} n}\left[\operatorname { c o s } \left(\theta_{\mathrm{a} n}-\right.\right. \\
\left.\left.\theta_{\mathrm{b} n}\right)+\cos \left(\theta_{\mathrm{a} n}+\theta_{\mathrm{b} n}\right)\right] \\
P_{12}=\frac{1}{2} U_{\mathrm{m} n} \mathrm{I}_{\mathrm{m} n}\left[\sin \theta_{\mathrm{a} n} \sin \theta_{\mathrm{b} n}+\right. \\
\left.\sin \left(\pi+\theta_{\mathrm{a} n}\right) \sin \left(\pi+\theta_{\mathrm{b} n}\right)\right]=P_{11} \\
\mathrm{P}_{13}=\frac{1}{6} U_{m n} I_{m n}\left[\sin \theta_{a n} \sin \theta_{b n}+\sin \left(\frac{2}{3} \pi+\theta_{a n}\right) \sin \left(\frac{2}{3} \pi+\theta_{b n}\right)\right. \\
\left.+\sin \left(\frac{4}{3} \pi+\theta_{a n}\right) \sin \left(\frac{4}{3} \pi+\theta_{b n}\right)\right] \\
=\frac{1}{2} U_{m n} I_{m n} \cos \left(\theta_{a n}-\theta_{b n}\right) \\
P_{13}=\frac{1}{6} U_{\mathrm{m} n} \mathrm{I}_{\mathrm{m} n}\left[\sin \theta_{\mathrm{a} n} \sin \theta_{\mathrm{b} n}+\right. \\
\sin \left(\frac{2}{3} \pi+\theta_{\mathrm{a} n}\right) \sin \left(\frac{2}{3} \pi+\theta_{\mathrm{b} n}\right)+ \\
\left.\sin \left(\frac{4}{3} \pi+\theta_{\mathrm{a} n}\right) \sin \left(\frac{4}{3} \pi+\theta_{\mathrm{b} n}\right)\right]=\frac{1}{2} U_{\mathrm{m} n} \mathrm{I}_{\mathrm{m} n} \cos \left(\theta_{\mathrm{a} n}-\theta_{\mathrm{b} n}\right)
\end{gathered}
$$

When $\mathrm{N} \geq 3, \mathrm{P}_{1 \mathrm{~N}}=\mathrm{P}_{0 \mathrm{~N}}$, and the dot product sum error quickly converges to zero. It can be seen that for the nth harmonics, as long as it ensures that the subharmonics sample 3 points or more in one cycle, the exact value of the subharmonic active energy can be obtained by the dot product sum method, and the corresponding periodic sampling point of the fundamental wave is $3 \mathrm{n}$. That is, for the nth harmonic, as long as the sampling rate of the power meter satisfies $f s \geq f_{0} * 3 n\left(f_{0}\right.$ is the fundamental frequency), accurate active power or active power energy can be obtained.

\subsubsection{Different Degrees of Harmonic Influence on Experiments}

A harmonic test was performed on a $0.5 \mathrm{~s}$-class power meter with a sampling rate of $3.2 \mathrm{kHz}$, a high-stability power source capable of generating 100 harmonics was used as a harmonic generation device. The error results obtained by comparing the power value measured by the power meter with the standard value output by the power source are shown in Figure 4 , and the data is shown in Table 2.

Table 2. Error test results of different harmonic power.

\begin{tabular}{lllll}
\hline Harmonic degrees & $\mathbf{1 6}$ & $\mathbf{2 1}$ & $\mathbf{2 4}$ & $\mathbf{3 1}$ \\
\hline Active power standard value / W & 28.994 & 28.994 & 28.994 & 28.994 \\
Active power measured value / W & 28.981 & 28.984 & 28.887 & 28.721 \\
Error/\% & -0.045 & -0.034 & -0.369 & -0.942 \\
\hline
\end{tabular}




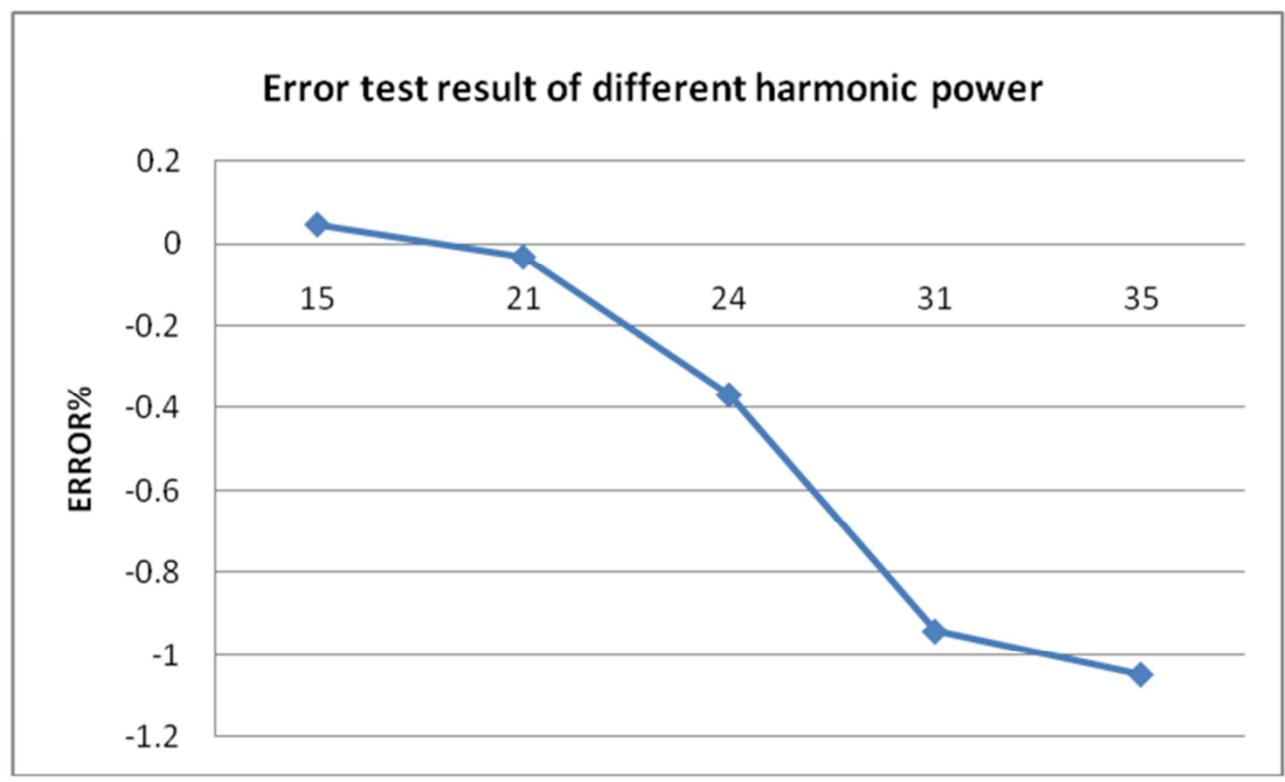

Figure 4. Error test result of different harmonic power.

Since the actual sample rate of the power meter is $3.2 \mathrm{kHz}$, the maximum accurate degrees of harmonic is:

$$
n \leq 3200 /(50 * 3) \leq 21.33
$$

It can be seen from Table 2 that the error is based on the 21 st harmonic as the demarcation point, and the harmonic errors less than or equal to 21 degrees are very small, and the 21 st harmonic error is $-0.034 \%$, which can be accurately measured. The harmonic errors greater than 21 degrees rapidly increase, in which the 24th harmonic error reaches $-0.369 \%$, and the error increases linearly with the degree of harmonics. The experimental results are consistent with the maximum degree of harmonics that the power meter can measure accurately.

\subsubsection{Grid Harmonics Component Field Test Data}

The Fluke-434 power quality tester was used to record the changes of the harmonic current at the grid point at the 50-MVA photovoltaic power station in Qinghai. The harmonic content of the grid-connected current is shown in Figure 5.

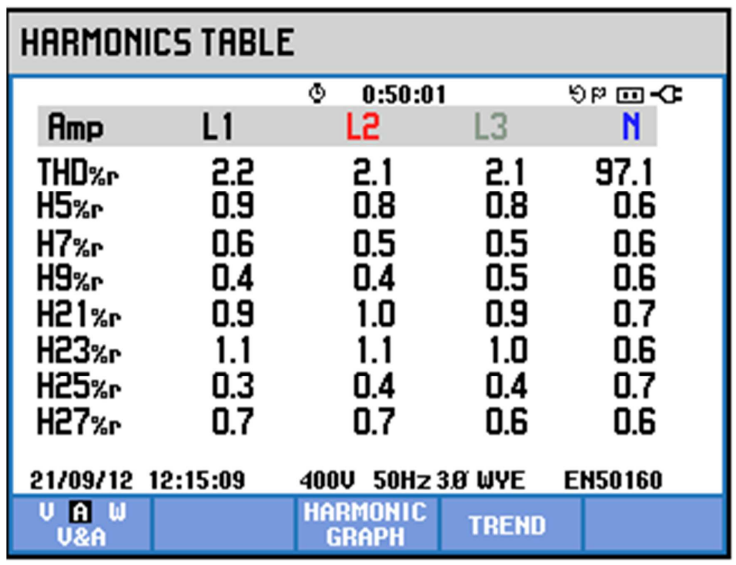

Figure 5. Harmonic contents in grid-connected current.
From Figure 5, we can see that the harmonic current in grid-connected harmonics include the $21 \mathrm{st}, 23 \mathrm{rd}, 25 \mathrm{th}$, and 27 th harmonics respectively, and the maximum number of harmonics is 27 degrees. We can easily calculate that the sampling rate fs of the power meter in the grid-connected harmonic environment must satisfy:

$$
f_{\mathrm{S}}^{\prime} \geq 50 * 3 * 27 \geq 4.05 \mathrm{kHz}
$$

Taking a high accuracy gate power meter as an example, the sampling rate is $12.8 \mathrm{kHz}$, the number of sampling points per week is 256 , and the maximum number of accurate harmonic degree is 85 . The accurate measurement of the harmonic components of the distributed power grid can be performed at an industrial frequency of $50 \mathrm{~Hz}$.

\subsection{Hardware Phase Matching Technique Solves the Impact of Grid Harmonic Phase Offset}

In Section 4.1 above, the accurate harmonic measuring we described is in the case of normal signal sampling, but in practice for distributed power grid harmonic signals, after the pre-circuit and ADC conversion, different phase shift $\Delta \varphi_{u}$ and $\Delta \varphi_{i}$ will be generated, resulting in additional phase difference between the signal entering the digital arithmetic unit and the original signal, ie:

$$
\varphi^{\prime}=\varphi+\Delta \varphi=\varphi_{u}-\varphi_{i}+\Delta \varphi_{u}-\Delta \varphi_{i}
$$

In order to ensure the accuracy of the measurement results, it is usually necessary to correct the additional phase difference $\Delta \varphi$, and the software shift method can be used to obtain a good calibration effect under sine conditions. However, if the load waveform contains harmonic components, the shift method will produce a linear phase shift effect. For nth harmonics, there will be a phase shift of $n^{*}|\Delta \varphi|$ leading to a larger error, and as the number of harmonics increases, the measuring error will also increase. 
Therefore, in order to minimize the higher harmonic measuring error and make it within an acceptable range, it is a feasible method to reduce $\Delta \varphi$ through the circuit design. The phase matching technology is a new technology used to achieve this goal.

The key of the phase matching technology lies in adjusting the voltage pre-circuit and the current pre-circuit parameters. The voltage pre-circuit diagram of voltage and current are shown in Figure 6 and Figure 7, respectively, so that the additional phase difference generated by the pre-units of the two circuits of voltage and current is as much as possible, namely:

$$
\Delta \varphi_{u}=\Delta \varphi_{i}
$$

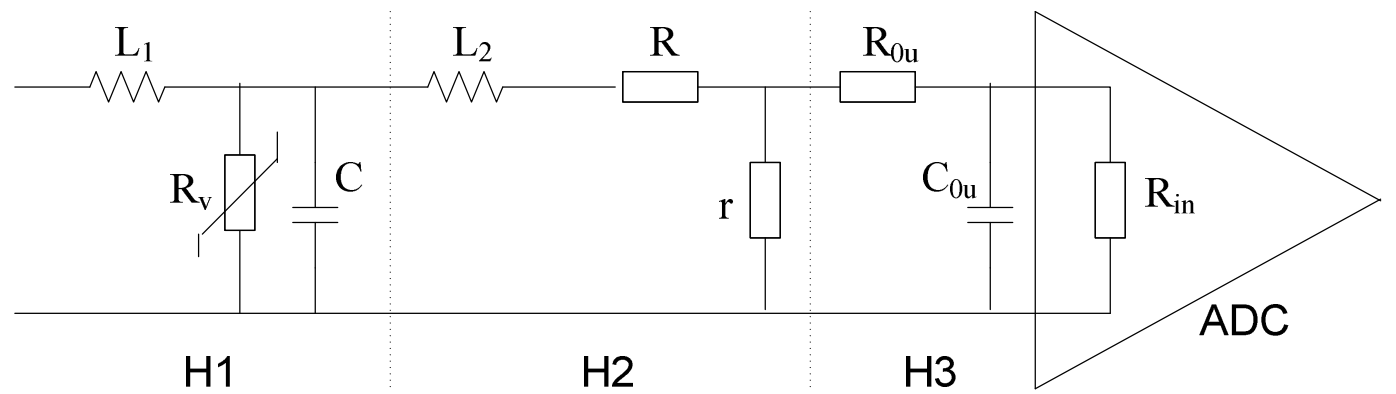

Figure 6. Typical voltage front-end circuit.

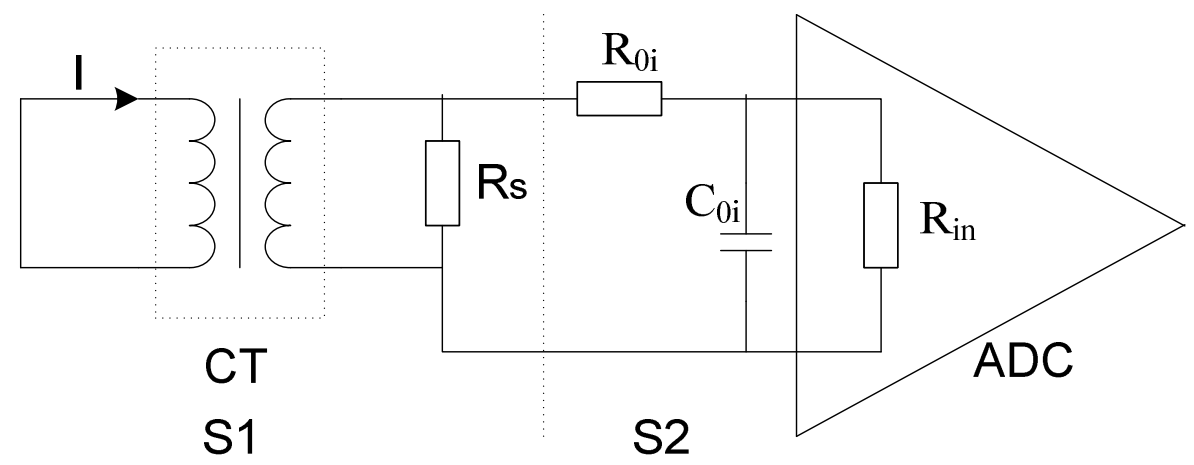

Figure 7. Typical current front-end circuit.

It can be seen that, under the influence of distributed power grid-connected harmonics, the use of hardware phase matching can effectively reduce the phase difference caused by the pre-circuit, so that the voltage and current as a whole phase synchronization can be achieved, thus ensuring accurate measurement of grid-connected harmonics.

\section{Measuring Analysis of Low Power Factor}

Under normal power generation conditions, the distributed power factor is above 0.9 . When the power is not generated or the power generation is low, the power factor is low. Taking a photovoltaic power station as an example, at night or on cloudy days, the input current of the power meter is less than $0.05 \mathrm{In}$ and the power factor is less than 0.1 . The conventional
Once the current sensing unit has determined the CT model, its phase shift characteristics cannot be adjusted; $\mathrm{The}_{1} \mathrm{H}_{1}$ and $\mathrm{H}_{2}$ circuits in the voltage pre-circuit have little influence on the phase shift characteristics of the overall circuit. Only by adjusting the $\mathrm{H}_{3}$ and $\mathrm{S}_{2}$ circuit parameters, namely adjusting $\mathrm{R}_{0 \mathrm{u}}, \mathrm{C}_{0 \mathrm{u}}, \mathrm{R}_{0 \mathrm{i}}$, and $\mathrm{C}_{0 \mathrm{i}}$, can the phase matching requirements be achieved.

For example, when $\mathrm{C}_{0 \mathrm{u}}=1000 \mathrm{pF}$, the additional phase difference of the measured voltage and current circuit is about $-0.23^{\circ}$, and when the adjustment circuit parameters making $\mathrm{C}_{0 \mathrm{u}}=100 \mathrm{pF}$, the additional phase difference $\Delta \varphi$ is reduced to $-0.04^{\circ}$. 
before delivery, and there is a phase adjustment deviation $\delta$ between the phase trim value and the actual value of the pre-circuit. Due to the influence of the phase adjustment deviation $\delta$, the power meter active power Pm and reactive power Qm are as follows:

$$
\begin{gathered}
\mathrm{P}_{m}=U I \cos (\theta+\delta) \\
\mathrm{Q}_{m}=U I \sin (\theta+\delta)
\end{gathered}
$$

Calculate the power meter active power error EP and reactive power error EQ according to the power meter error calculation formula as follows:

$$
\begin{aligned}
& E_{\mathrm{P}}=\frac{\mathrm{P}_{\mathrm{m}}-\mathrm{P}}{\mathrm{P}}=\frac{U I \cos (\theta+\delta)-U I \cos (\theta)}{U I \cos (\theta)} \\
& =\cos (\delta)-\sin (\delta) \operatorname{tg}(\theta)-1 \\
& E_{Q}=\frac{\mathrm{Q}_{\mathrm{m}}-\mathrm{Q}}{\mathrm{Q}}=\frac{U I \sin (\theta+\delta)-U I \sin (\theta)}{U I \sin (\theta)} \\
& =\cos (\delta)-\sin (\delta) \frac{1}{\operatorname{tg}(\theta)}-1
\end{aligned}
$$

Phase adjustment deviation $\delta$ is generally less than 2 points, and $\cos (\delta)$ is approximately equal to 1 . The error formula is simplified as follows:

$$
\begin{gathered}
E_{\mathrm{P}} \approx-\sin (\delta) \operatorname{tg}(\theta) \\
E_{Q} \approx \sin (\delta) \frac{1}{\operatorname{tg}(\theta)}
\end{gathered}
$$

From the error formula, it can be seen that the lower the power factor is, the larger the $\operatorname{tg}(\theta)$, and the greater the effect of $\delta$ on the active error, the smaller the influence on the reactive power error. On the contrary, the higher the power factor is, the larger the $\operatorname{tg}(\theta)$, and the smaller the influence of $\delta$ on the active error, the greater the influence on reactive power error.

Due to the non-linearity of the angle difference of the current transformer, the phase adjustment deviation $\delta$ of the power meter under different current is different, and the rated current point is the best, and the small current point is poor. The 0.01 degree rated current point angle difference of a 0.05 class conventional current transformer is 2 to 4 points larger than the rated current point angle difference. Taking 3 points as an example, the error of active power and reactive power of the power meter when calculating the power factor at the minimum current of 0.1 and 0.9 respectively are shown in Table 3.

Table 3. Small-current active and reactive error under different power factors.

\begin{tabular}{llll}
\hline Phase deviation (') & Power factor & Active power error (\%) & Reactive power error (\%) \\
\hline \multirow{3}{*}{3} & 0.1 & -0.868 & 0.009 \\
& 0.9 & -0.0423 & 0.18 \\
\hline
\end{tabular}

From Table 3, it can be seen that the active power metering accuracy under low power factor is greatly affected, and the reactive power metering accuracy under high power factor is greatly affected.

The angle difference of the current transformer stems from the fact that the magnetizing current must be used to establish the induced electromotive force during operation. Otherwise, there is no magnetic flux in the iron core. The primary and secondary currents cannot transmit energy and the current sensor cannot work, which is shown in Figure 8.

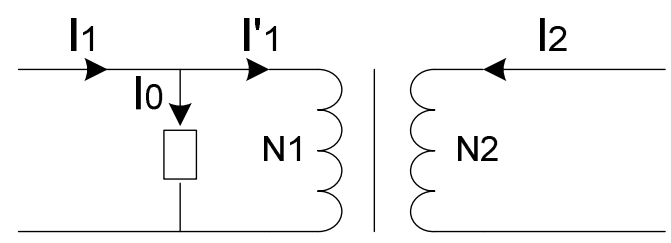

Figure 8. Current transformer working principle.

At this time, the vector relationship between the magnetic potentials is shown in Figure 9.

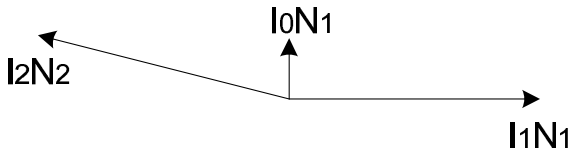

Figure 9. Magnetic potential vector with magnetizing current.

It is easy to see that due to the existence of the magnetizing magnetic potential, the primary and secondary current no longer follow a strict proportional relationship, and the phase difference between them is not strictly $180^{\circ}$, that is, the angle difference is zero. When the current I1 is larger, the influence of the magnetizing current I0 is smaller, and the angle difference between I1 and I2 is also smaller; When I1 is smaller, the effect of I0 is significant, causing the linearity of the transformer in the small current segment to decrease, and the angular difference increases.

In order to make up for this deficiency in the sensing principle of CT, a completely new current sensing technology, zero-flux current sensing technology, is used. The so-called "zero flux" means that the flux in the iron core is reduced to an extremely low approximately zero state by an external compensation method, so that the current sensor achieves very high accuracy.

A 0.3 (6) A zero flux CT test results are shown in Table 4. 
Table 4. Zero-Flux CT Angle Difference Test Data.

\begin{tabular}{ccllllll}
\hline \multicolumn{2}{l}{ Zero flux CT angle difference } & & & & & \\
\hline & Measuring point & $\mathbf{0 . 0 1 I n}$ & $\mathbf{0 . 0 5}$ In & $\mathbf{0 . 1}$ In & In & 10 In & 20 In \\
\hline $1 \#$ & Angle difference (') & 0 & -0.05 & -0.06 & 0.02 & 0.04 & 0.04 \\
$2 \#$ & Angle difference (') & 0.19 & 0.09 & 0.08 & 0.09 & 0 & 0.18 \\
$3 \#$ & Angle difference (') & 0.04 & -0.1 & -0.11 & 0 & -0.05 & -0.09 \\
$4 \#$ & Angle difference (') & 0.1 & -0.02 & -0.05 & 0.03 & -0.04 & -0.3 \\
\hline
\end{tabular}

It can be seen that using a zero-flux current transformer can effectively reduce the phase adjustment error of the power meter at each current point, and improve the measuring accuracy of active and reactive power.

\section{Conclusion}

This article analyzes the effects of bidirectional wide range measuring, DC components, and harmonic components on energy metering via analyzing the distributed power grid-connection. Analyze the field power meter data of the wind farm to analyze that a wide-range measuring gateway can accurately measure the reverse power; By analyzing the influence of the secondary transformer on the DC component, the effect of the DC component on the energy metering is almost negligible when the distributed power grid connection is injected with the $0.5 \% \mathrm{DC}$ current component. By analyzing the dot product sum harmonic wave algorithm, it is concluded that the harmonics of the distributed power grid affect the accurate sampling rate of the power energy, and the phase-matching technology is used to solve the synchronous sampling problem of voltage and current under the influence of harmonics. By analyzing the factors that affect the measuring accuracy under different power factors, it is concluded that the use of zero-flux current transformers can effectively improve the measuring accuracy.

\section{References}

[1] Si-Hun Jo, SeoEun Son, and Jung-Wook Park, "On improving distortion power quality index in distributed power grids," IEEE Trans. Smart Grid, vol. 4, no. 1, pp. 586-595, Mar. 2013.

[2] Tao Zhou and Bruno François, "Energy management and power control of a hybrid active wind generator for distributed power generation and grid integration" IEEE Trans. Ind. Electron., vol. 58, no. 1, pp. 95-104, Jan. 2011.

[3] KANG Chenggong, LI Xianwei, ZHANG Guojun. Research on power quality control strategy incorporating distributed generation. Power System Protection and Control. 2016, 44 (16):99-105.

[4] V. F. Corasaniti, M. B. Barbieri, P. L. Arnera, and M. I. Valla, "Hybrid active filter for reactive and harmonics compensation in a distribution networks," IEEE Trans. Ind. Electron., vol. 56, no. 3, pp. 670-677, Mar. 2009.

[5] M. Prodanovic and T. C. Green, "High-quality power generation through distributed control of a power parkmicrogrid," IEEE Trans. Ind. Electron., vol. 53, no. 5, pp. 1427-1436, Oct. 2006.

[6] Peng Cheng. Research and design of bidirectional power measurement and harmonic detection system based on DSP. Guangxi University, 2017.

[7] Wang Mengwei, Yan Yang. Analysis of bidirectional power energy measurement for distributed generation [J]. Electrotechnics Electric, 2013, (5): 15-17, 22.

[8] Zhao Kangshen, Wang Qingzhang, Guo Tianyong, et al. FPGA-based power flow analysis of photovoltaic grid-connected power generation system and the research of dual power measurement [J]. Acta Scientiarum Naturalium Universitatis Nankaiensis. 2009, 42 (3): 22-28.

[9] Tao Weiqing, Li Jiaxi, Ding Ming, Wang Leqin, Gu Zhixia. Development and Comparison on Standard for Interconnecting Distributed Resources with Electric Power Systems. JOURNAL OF ELECTRICAL ENGINEERING. 2016, 11 (4):1-8.

[10] Q/GDW 666-2011. Test technical specification for distributed resources connected to distribution network [S].

[11] Bao Wei, Hu Xuehao, He Guoqing, et al. Study on standard for grid-integration of distributed resources [J]. Power System Technology, 2012, 36 (11): 46-52.

[12] Fu Xueqian, Chen Haoyong, Liu Guote, et al. Power quality comprehensive evaluation method for distributed generation [J]. Proceedings of the CSEE, 2014, 34 (25): 4270-4276.

[13] Q/GDW480-2010. Technical rule for distributed resources connected to power grid [S].

[14] Tian Xiaoqian. Research on Current Transformer and Energy Metering Affected by DC Bias [D]. Baoding: North China Electric Power University, 2014.

[15] Tan Bingyuan, Lu Jiping, Jiangbo et al. Analysis on Performance of Current Transformer Affected by DC Bias [J]. Power grid technology, 2014, 38 (5): 1408-1413.

[16] Wang Huan, Mao Anlan, Wang Quan, et al. Research on the error characteristics of measurement for CT under DC magnetic bias $[\mathrm{J}]$. Process Automation Instrumentation, 2014, 35 (6): 47-53.

[17] Wei Gang, Wu Weili, Hu Danyun, et al. Distributed generation and effects of its parallel operation on power system [J]. High Voltage Engineering, 2007, 33 (1): 36-40.

[18] Yao Li. The method of electric energy measurement under the influence of harmonics [J]. Electrical Measurement \& Instrumentation, 2005, 42 (10): 20-23, 10. 


\section{Biography}

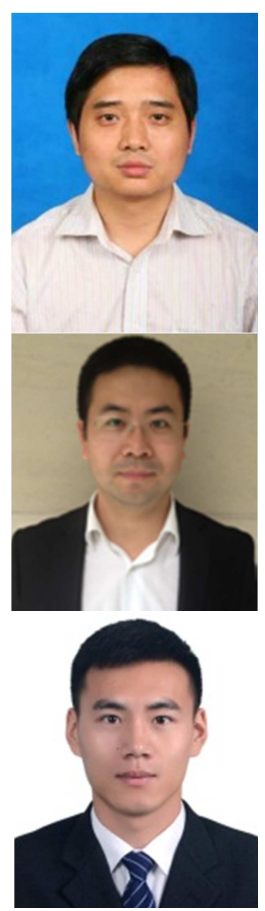

Li Pengcheng (1975-), male, Yinjiang, Guizhou, master, senior engineer. The main research direction is electrical energy metering.

Zhao Yantao (1981 -), male, engineer, Research on measuring technology of electric energy meter and electric energy measurement.

Cong zhongxiao (1990-), male, Weihai, Shandong, Master graduate student, engineer, the main research direction is electric energy measurement, power system operation and control.

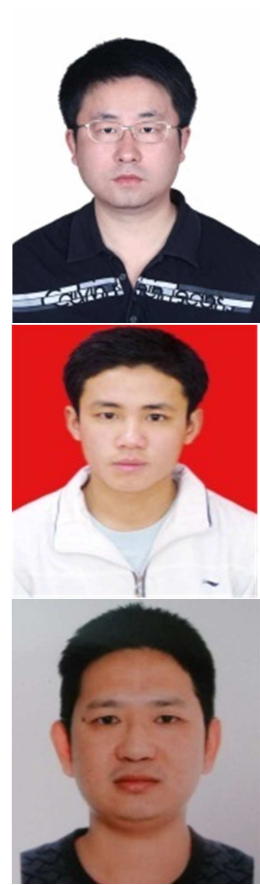

Zhao Yunbin (1984-), male, Guiyang, Guizhou, graduate student, senior technician. The main research direction is electrical energy measurement.

Xu Hongwei (1985-), male, Guiyang, Guizhou, Master graduate student, senior engineer. The main research direction is electrical energy measurement.

Su Yuhua (1978 -), male, engineer, Research on measuring technology of electric energy meter and electric energy measurement. 